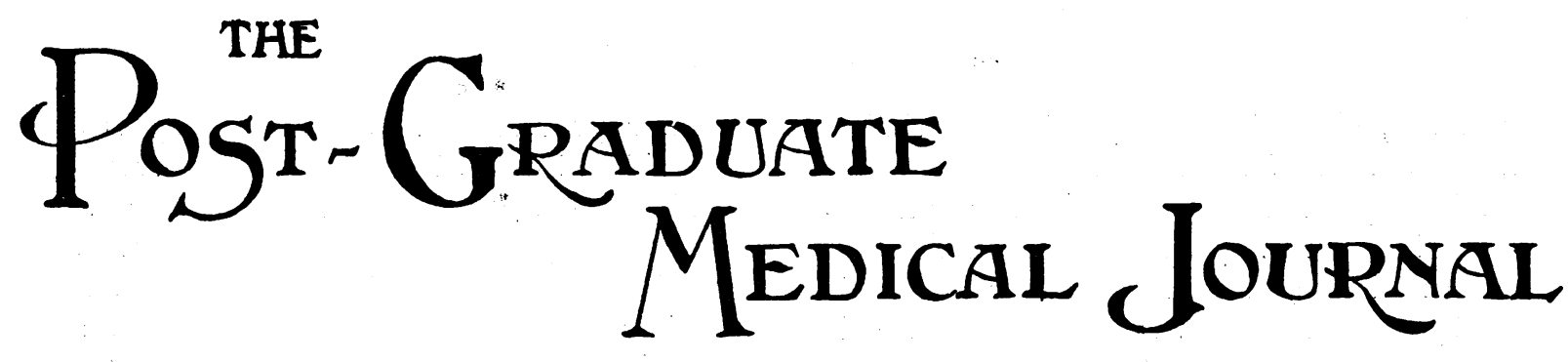

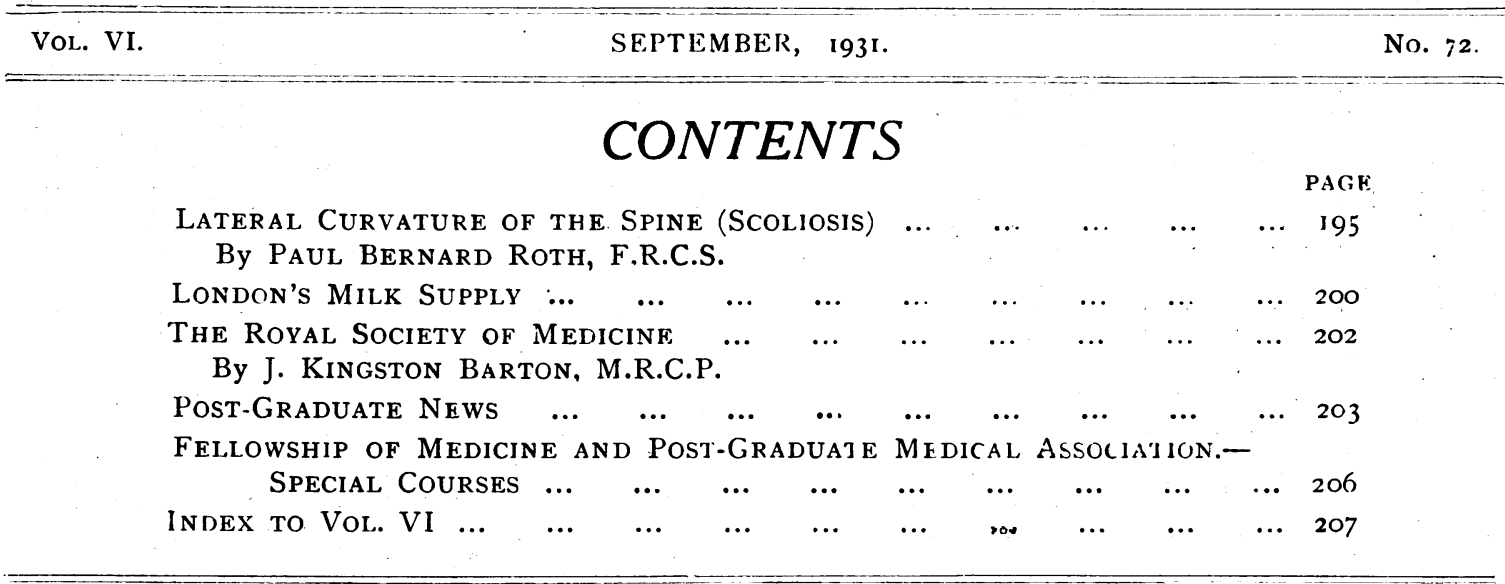

\section{LATERAL CURVATURE OF THE SPINE (SCOLIOSIS).}

\section{By PAUL BERNARD ROTH, F.R.C.S.}

WHEN a child begins to hold herself badly, stooping over her lessons, slouching at meals, when her mother is telling her continually to sit up straight, she is described as suffering from stoop or rouild shoulders, these terms being synonymous.

Such a condition may or may not lead on to lateral curvature, but one never finds lateral curvature present without a certain amount of stoop. When there is lateral curvature it is customary to label the case as such, and the stoop is regarded as a subsidiary symptom and allowed to fall into the background. But it is there all the time and has to receive treatment along with the lateral curvature.

In the following description this should be borne in mind.

\footnotetext{
1 This paper is based on a demonstration given to the members attending a Post-Graduate Course at the Miller General Hospital in July, 1931.
}

General Canses. - The cause of these con. ditions is a weakness of the whole of the structures of the body-bones, muscles, fasciæ, ligaments, \&c., a condition which is very well described by the term "skeletal insufficiency," combined in the majority of cases with long-continued sitting and standing in stooping or relaxed positions, such as standing on one leg, sitting writing, reading or sewing with the trunk twisted to one side or with the thighs crossed. The position of writing as generally practised is specially to be blamed; while rapid growth, onset of puberty and unsuitable clothing are contributory causes.

Some of the worst cases of scoliosis follow an attack of infantile paralysis affecting the spine, which produces "skeletal insufficiency" in its most aggravated form.

American surgeons prefer "intrinsic muscle imbalance" to "skeletal insufficiency" : but this presupposes that it is the muscles only which are at fault.

\section{DEFINITION.}

Lateral curvature of the spine, or scoliosis, is an acquired deformity occurring in 
children, characterized by lateral deviation and rotation of the spinal column, accompanied by alteration in the normal anteroposterior curves. [Congenital malformation of the spine, where a half vertebra is interposed between two other vertebræ, is an altogether different condition, and is not being considered here.]

\section{VARIETIES AND DEgReEs.}

The cases can be divided into three groups :-

Type I, where the deformity is entirely postural.

Type II, where it is partly postural and partly structural.

Type III, where it is entirely structural.

But I myself find it more convenient to divide them into two groups, the postural and the structural, remembering that in a structural case much of the deformity may be postural. In all cases the deformity begins as a postural one, and only after it has persisted for some time does it become a structural one.

This division into two classes is important, because the postural cases are entirely curable, whereas the structural cases are only partly so.

\section{Structural Changes.}

The first change is in the intervertebral disc ; it becomes wedge-shaped. Then the body (centrum) follows suit, the apex of the wedge being directed towards the side of the concavity. The wedging is due to pressure preventing growth.

As time goes on the transverse process on the side of the convexity projects more and more backwards and less and less outwards, the transverse process on the side of the concavity projecting more and more forwards. The vertebral aperture becomes altered in shape. Normally it is like a circle with the upper and lower poles slightly flattened. In the scoliotic vertebra it is egg-shaped, the broad end of the egg corresponding to the convexity. The spinous processes are deviated away from the middle line on the side of the convexity, $\underset{\mathbb{D}}{z}$ but in twisting they lag behind so that the tip of the process may actually point towards the side of the concavity. The reason for $\stackrel{0}{?}$ this is that the rotation takes place round an axis situated at a point in space about an $\overline{\bar{n}}$ inch posterior to the tip of the spinous $\frac{\widehat{\phi}}{\alpha}$ process. A severe rotation of the vertebral bodies can take place while still the tips of the $\vec{\circ}$ spinous processes seen under the skin appear very little displaced from the middle line. $\vec{\sigma}$ This is well shown in the model which I $\vec{\circ}$ have here.

In addition, changes take place in the bony structure of the bodies. A vertebral body $N$ consists of an upper and lower plate, $\vec{\oplus}$ separated by a mass of spongy bone. The arch is attached chiefly to the upper plate. $\rightarrow$ When rotation takes place the upper plate $\mathcal{D}_{\mathbb{D}}$ with the arch is twisted on the lower plate, $\underset{\mathbb{D}}{\mathbb{D}}$ so that the fibres or spicules in the spongy $\frac{3}{\sigma}$ bone which normally pass vertically between $\frac{\Phi}{?}$ the upper and lower plates are made to pas obliquely. Lorenz has described this by. saying that "the torsion of the scoliote vertebral column takes place in the osseous tissues of the vertebral column itself."

Changes in the Ribs.-Owing to the lateral $\frac{0}{\circ}$ deviation the ribs on the concave side get $\underset{\vec{F}}{\vec{P}}$ pressed together, and those on the convex 3 side become separated. Owing to the rotation of the vertebra, as already stated, the transverse process on the convex side is directed more backward. As the vertebral:end of the rib is attached to the transverse $\stackrel{3}{\text {. }}$. process it must follow it, and so the angle of $\varnothing$ the rib is made sharper on the convex side. On the concave side the opposite occurs:? the transverse process is directed more forwards, carrying the vertebral end of the rib with it, and thus the angle is flattened ${ }^{\circ}$ out. In front the same bulging and flattening are produced, but on opposite sides. Thus, if the right ribs are prominent behind? the left ribs are prominent in front. The bulging of the ribs on one side behind,,causes the scapula on that side to project $\frac{0}{0}$ 
more than the other: the bulging of the ribs in front causes the breast on that side to project more than the other : and often what first calls a parent's attention to a lateral curvature is a prominence of one shoulderblade or of one breast.

Changes in the Loin.-A bulging of the erector spinæ is found in the lumbar region on the convex side, caused by the projection backwards of the transverse processes : on the concave side there is a flattening.

Changes in the Chest.-In consequence of the changes in the ribs the shape and capacity of the chest are altered. The diagonal diameter from, say, the right ribs behind to the left ribs in front-i.e., from the bulge behind to the bulge in front-is increased: and the corresponding one on the other side-i.e., from the flattening behind to the flattening in front-is diminished.

The chest capacity is diminished, especially the half on the convex side, for the ribs being more sharply curved enclose a smaller area. On the concave side, the ribs being less curved, the area horizontally is greater, but here the height is diminished. Reduced lung capacity is one of the serious effects of scoliosis.

Changes in the Ligaments and Muscles.These take part in the lateral deviation and rotation, and accommodate themselves to the changes in the bones and discs.

It has been suggested by one or two writers that it is the ribs which become deformed first, and that it is these which distort the spine. One could not have a better example of what is known as "putting the cart before the horse."

When there is structural deformity, its existence is recognized by the fact that when the patient completely flexes the spine, instead of the two halves of the trunk on either side of the spinous processes being equal, one or other is seen to be more prominent, see fig. $2 a$. The amount of this prominence, which can be accurately recorded, is taken as a measure of the degree of structural deformity present, and it is described as being slight, moderate, severe, or extreme. There are other methods of measuring and recording it, Dr. J. K. Young has described sixty-seven, but this is so simple and so accurate that I have never seen the necessity for any other.

Six varieties of deformity are met with, which may be described as follows, viewing the spine from behind :-

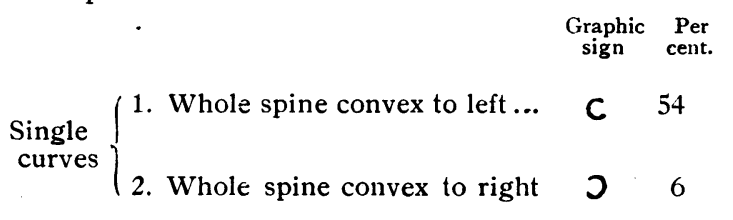

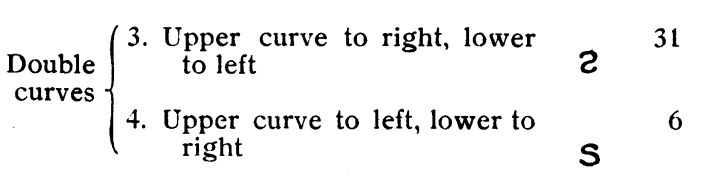

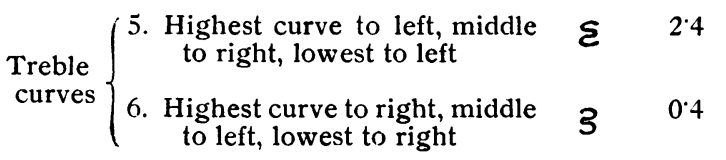

One often hears a case described as serious "because there are two curves": but this is confusing the variety of the curve with its severity.

\section{Examination of the Patient.}

(I) Measure the legs; if one be shorter than the other, equalize matters by placing a suitable block beneath the foot of the short limb before proceeding further.

(2) View the spine from behind, with the patient stripped.

Observe the "habitual position" of the patient, and note if one or more signs of scoliosis are present. These are : an elevation of one shoulder, an enlargement of one arm space (the space between the trunk and the arm as it hangs down), a prominence of one hip, a lateral deviation of the line of the spinous processes, an abnormal projection of one scapula, an obvious bulging of the ribs on one side.

Observe the patient standing with the feet 
together and knees straight, spine fullysflexed, the head and arms hanging down limply (see fig. 2a). On looking at the "horizon" of the back at different levels any structural deformity will be at once noticed; the ribs in the dorsal region or the erector spinæ in the lumbar region, will project more on one side than the other, due to rotation of the bodies of the vertebræ. If there be no such projection, the case is one of postural scoliosis; if there be projection, structural scoliosis is present, and the amount of it should be at once recorded by using a malleable tin tape. This is moulded across the ribs (see fig. $2 b$ ), and loins at certain welldefined levels, and a tracing of it made on paper. This is cut out with scissors and the paper "positive" thus obtained pasted in the case-book, with the date and the patient's name and any other particulars of importance. If the patient be seen years later, one can discover by taking a similar moulding whether the deformity has lessened, remained stationary, or increased.

(3) View the Patient from the Side.Observe the state of the antero-posterior curves; they are usually exaggerated. The dorsal curve, with concavity forwards, is increased ; the patient stoops and pokes the head forwards. To bring back the centre of gravity to the middle of the body, compensatory increase of the normal lumbar lordosis develops, which makes the abdomen prominent. With the stoop there is a drooping downwards and forwards of the scapulæ.

(4) View the Patient from in Front. Observe the degree of development of the chest, the lung capacity, the presence of a bulge in the ribs on one side or the other, the prominence of a breast or of a hip.

Prognosis. - Having observed the "habitual position" in which the patient stands, it is necessary to find the "best possible position" in which she can be placed. This will show at once what improvement can be brought about, for she can be trained to maintain this position always. When a case with even severe structural deformity is put in the "best possible position," often much of the deformity is hidden, and this is what the surgeon wants: for if there is structural deformity it cannot be got rid of.

(5) Look at the Clothes.-These must leave plenty of room across the chest when in the "best possible position." The stockings must not be attached to a bodice with straps passing over the shoulders, but kept up by a suspender-belt or by garters.

(6) Test the Vision.

(7) Test the Air-passages.

\section{TREATMENT.}

This will depend on the views of the surgeon.

The different methods at his disposal are:-

(I) Lying down for one or more hours $\vec{c}$ daily.

Comment.-This only makes the patient weaker.

(2) "Gradual correction" in a support of corset.

Comment.-No correction occurs.

(3) "Rapid correction" in a plaste jacket.

Coinment.-A bulging rib can be flattened out temporarily, but the deformity recurs again when the jacket is removed.

(4) Gymnastic methods.

Comment.-These give excellent results; the particular method advised is that known as "posture and exercise."

(5) Operative methods. Fusion operations, resection of bulges, \&c.

Comment.-To mention these is to con- $\frac{\delta}{3}$ demn them (see later).

\section{Treatment by Posture and Exercise.}

This consists in exercising the patient daily in the "best possible position," so that " gradually this position becomes the habitual one. This usually takes from two to three ${ }_{\sigma}^{\omega}$ months, and during this time the patient must stay away from school. No attempt is made to get rid of the structural deformity $\stackrel{?}{?}$ present, as it is realized that this is impossible. $\frac{0}{0}$ 


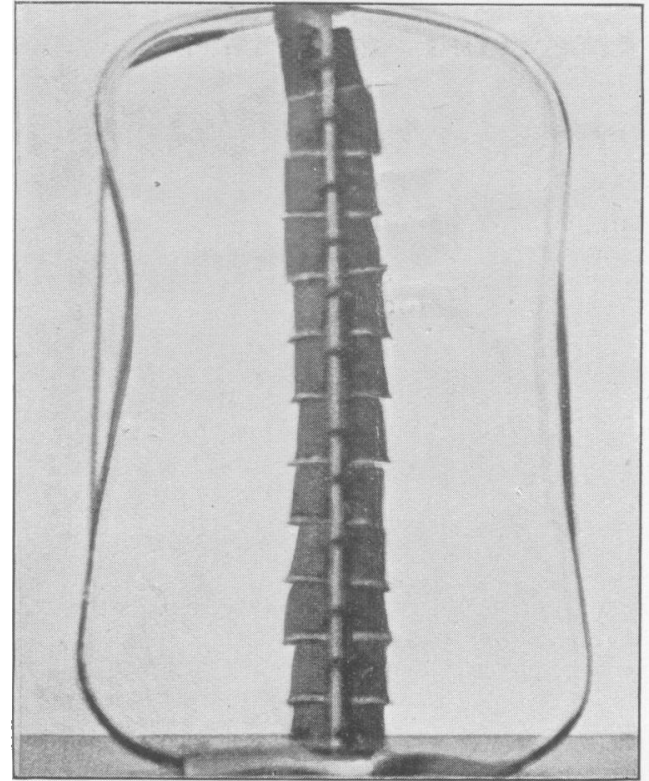

$a$

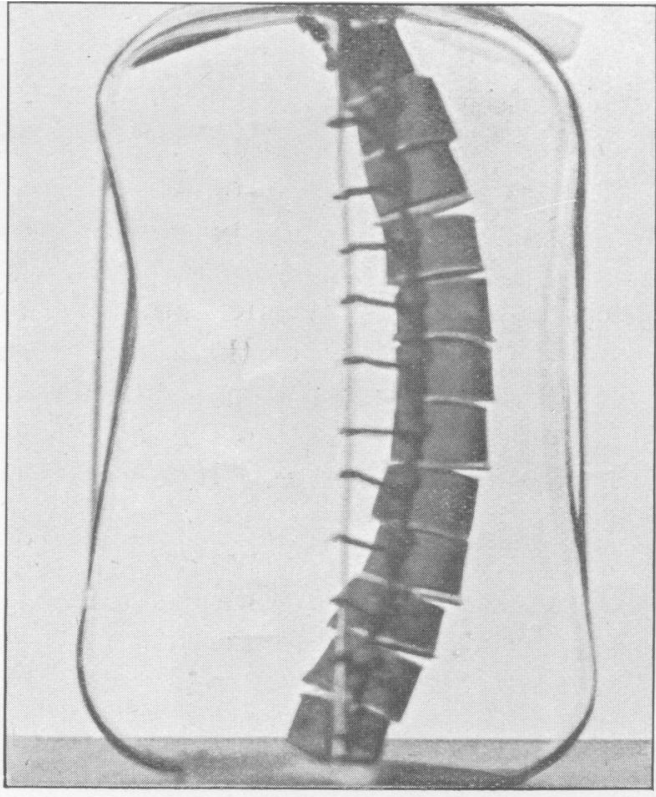

$b$

Fig. 1.- Photographs of a model of the dorsal vertebræ (bodies and spinous processes only), to show how a severe rotation of the vertebral bodies can take place while still the tips of the spinous processes seen under the skin appear very little displaced from the middle line. $(a)$ Spine straight; $(b)$ spine laterally curved. The tight string represents the supraspinous ligaments.

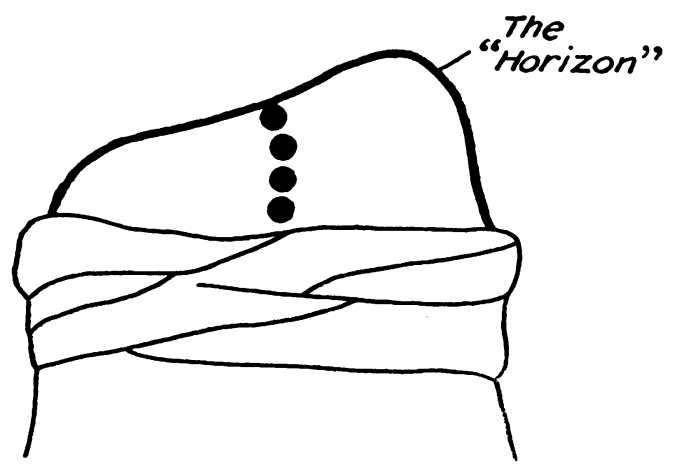

FIG. 2a.-The spine of a case of 2 -shaped scoliosis with severe structural deformity, sfanding with the spine fully flexed, seen from behind. Note the "horizon" of the back, and that though there is severe structural deformity, the spinous processes are only very slightly deviated from the mid-line.

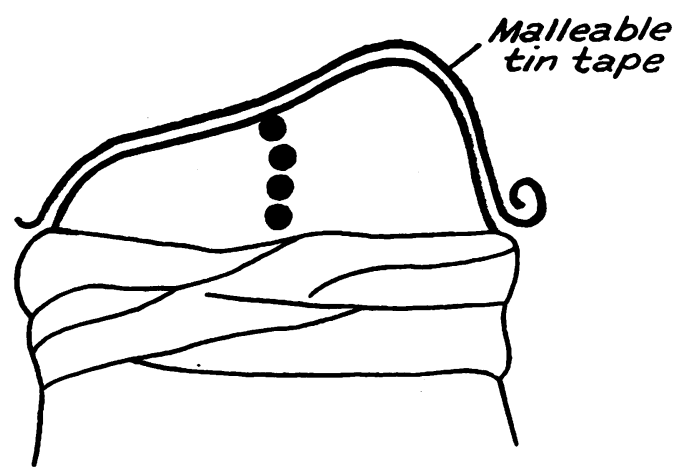

FI(i. 2b.-The same, showing how the amount of structural deformity may be recorded by using a malleable tin tape.

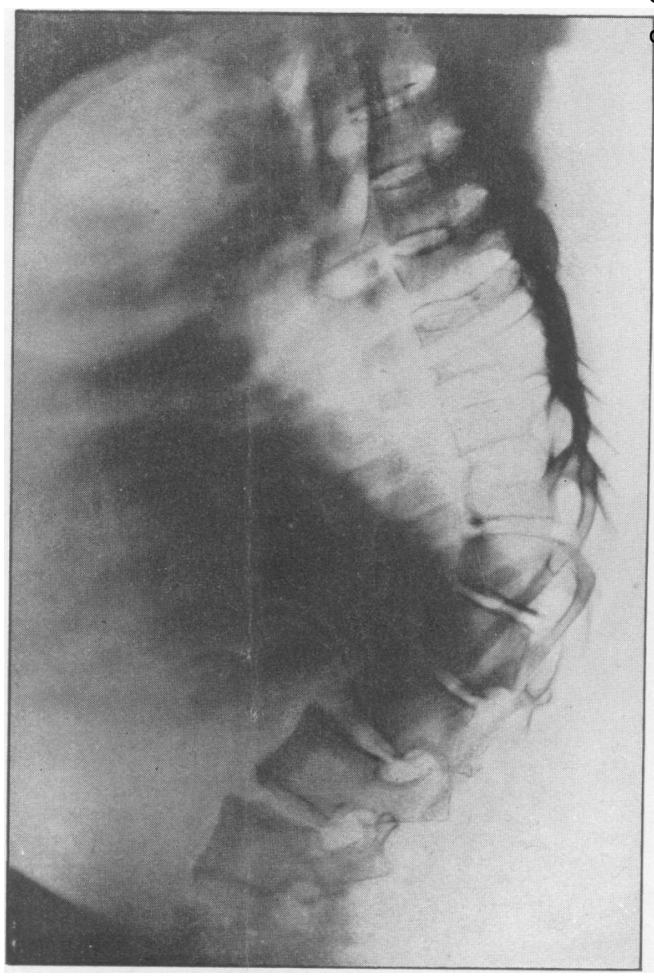

FIG. 3.-Spondylosis Juvenilis. Edith G., aged 18, "back sticking out in the middle, getting worse the last month." Radiogram, lateral view, showing wedging of $\varrho$ three vertebral bodies. 
As the patient begins to assume the "best possible position" always, all tendency to further crooked growing is abolished, and the increase in structural deformity entirely arrested. From observations made on I,000 cases treated by this method, it was found that structural deformity was entirely arrested in all but 5.5 per cent of cases.

The treatment of course gets rid of all postural deformity, and makes the patient in all but severe cases look perfectly straight. It abolishes all pain in the back, and greatly improves the patient's general health.

The treatment is carried out according to the following principles :-

(I) Attention to Dress.-If the clothes are not loose enough to allow of patients assuming the "best possible position," the efforts of the surgeon are largely wasted. It often happens that patients are exercised undressed-a quite unnecessary proceedingand are then allowed to dress again in their badly-fitting clothes, which at once cause them to assume the "habitual position," the very position the surgeon wants to avoid.

(2) Daily systematic strengthening, for from one to three months, of the spinal muscles, including development of the chest.

These exercises are all clone in the "best possible position" for the first month, regardless of the particular variety of curve present. After a month, the patient is examined again undressed, and a position of the arms decided upon which makes the spine look straightest. This is the so-called "keynote position." Thereafter many of the exercises are done in that position.

The exercises are done with the clothes on. After each exercise the child places herself in the "best possible position" against the wall, and then walks to her seat and sits down, still with her spine in that position, and so remains until called upon to do the next exercise.

At first the exercises are very easy, but gradually they are made more and more difficult as the strength of the patient increases. It is very important that no fatigue whatever should result from them. They consist largely of concentric and eccentric movements, patient resisting or surgeon resisting, but there is not time to describe them to-day.

The daily exercises, with rests in between, take about an hour.

(3) Daily re-education of the muscular sense, as to an erect or improved position.

A great many crooked patients when told to stand up straight place themselves in a worse position than before. They do not know what is straight. They must be shown over and over again until they learn.

(4) Maintenance of the improved position all day.-It is not sufficient to give the patient treatment each day for an hour, and then let her return home to sit and stand and walk in the same old bad position until the next visit for treatment on the following day. Steps must be taken to ensure as far as possible that correct postures are assumed always.

The patient should not stand at lessons, should have a comfortable back to lean against when sitting, and a suitable desk provided for writing. Far and away the best desk I have seen is that made by Hammer and Co., and known as the "hygienic adjustable single desk."

(5) Subsequent home treatment for many months to prevent relapse from the improvement or cure which has been obtained. This consists in going through a number of stated exercises daily, and in assuming the "best possible position" against the wall before and after every meal.

Note.-Position while Asleep. As the whole point of the treatment depends upon the voluntary muscular action of the patient, no possible benefit can arise by interfering with the position of the patient during sleep.

\section{DIAGNOSIS.}

There are two conditions which may be confused with scoliosis and stoop: one is tubercular disease of the spine (Pott's disease) and the other is an only recently and imper-

.


fectly described condition which I prefer to name spondylosis juvenilis.

In tubercular disease the earliest sign is rigidity; an X-ray shows caries of one or more vertebral bodies and bulging of the psoas sheath : when deformity occurs there is a projection backwards in the midline, and if pain be present movement makes it worse.

In spondylosis, which is also known as student's stoop, fived stoop, dorsal wedging round back, there is a very bad stoop, chiefly in the mid-dorsal region, which comes on insidiously, and which is quite incapable of being corrected in the slightest degree. An $\mathrm{X}$-ray, lateral view, shows that two or more vertebral bodies have become markedly wedge-shaped (see fig. 3).

\section{Note on an Operative Method of TREATMENT.}

I will conclude by drawing your attention to a paper which appeared in the Journal of Bone and Joint Surgery, for January, I93I. It is an account of 360 cases of scoliosis treated by the fusion operation of Russell A. Hibbs. The spinous processes from the lower cervical to the upper lumbar region are laid bare in one long incision, and split in such a manner that bony fragments from each vertebra are made to join with similar bony fragments of the vertebra above and below, the hoped-for result being one long bone joining all the operated on vertebræ together. This operation is supposed to arrest the onset of deformity. There were five deaths directly attributable to the operation. The oldest patient was 46, the youngest I year II months. Eighty-eight per cent. were operated upon when they were between Io and 20 years of age.

As a result of all these operations, $69^{\circ} 5$ per cent. of the cases showed no increase in deformity, while in $30^{\prime} 5$ per cent. it was worse. The results therefore are not nearly as good as the results obtained by " posture and exercise"; but what will happen to the child aged I year I I months when it grows up, with its spine fused in one long poker?

\section{LONDON'S MILK SUPPLY.}

TUBERCUlosis is an ever-present scourge in England, though of late years strenuous efforts have been made to reduce the high figures of incidence. One of the most important steps taken to effect this reduction has been the endeavour to raise the quality of the milk supply by the elimination of infected animals, by the use of scientific treatment to reduce the bacteria content, and by the observance of far higher standards of cleanliness. In view of the modern theory that increased consumption per head of milk means better national health, it may be realized that to maintain a high standard both of quality and of quantity is no small problem, and is one that is particularly difficult to solve when dealing with the huge area covered by London.

The population of London is in the neighbourhood of 9,000,000, and even at the absurdly low estimate of half a pint of milk per head, one can arrive at some idea of theo magnitude of the organization required to provide, at a reasonable price, a sufficient quantity of safe milk to supply such a demand. It is obvious that a population of such a size means that the distances to be covered by the milk supply before it reaches its final destination are enormous. In some cases local dairies in London make their own arrangements for the transport of the milk from farms to the shops, whence it is distributed in accordance with each dairy owner's fancy. In other cases there are big organizations which arrange for the collection of milk from affiliated farms, and from a central point in London distribute to the local dairies under their direct managementa direct management which, in fact, covers the whole journey of the milk from the cows to the customers. It is interesting to follow the complicated workings of an organization of this kind, with the many technical and administrative details to be considered in connection with such a perishable commodity as milk, especially when the organization has a high reputation to maintain. 